\title{
Natural chalcones as dual inhibitors of HDACs and NF-кB
}

\author{
B. ORLIKOVA ${ }^{1}$, M. SCHNEKENBURGER ${ }^{1}$, M. ZLOH ${ }^{2}$, F. GOLAIS ${ }^{3}$, M. DIEDERICH ${ }^{1}$ and D. TASDEMIR ${ }^{2,4}$ \\ ${ }^{1}$ Laboratory of Molecular and Cellular Biology of Cancer, Cancer and Blood Research Foundation, Kirchberg Hospital, \\ Luxembourg, Luxembourg; ${ }^{2}$ Department of Pharmaceutical and Biological Chemistry, School of Pharmacy, \\ University of London, London WC1N 1AX, UK; ${ }^{3}$ Comenius University, Faculty of Sciences, \\ Department of Microbiology and Virology, Bratislava, Slovakia
}

Received March 16, 2012; Accepted May 4, 2012

DOI: $10.3892 /$ or.2012.1870

\begin{abstract}
Histone deacetylase enzymes (HDACs) are emerging as a promising biological target for cancer and inflammation. Using a fluorescence assay, we tested the in vitro HDAC inhibitory activity of twenty-one natural chalcones, a widespread group of natural products with well-known anti-inflammatory and antitumor effects. Since HDACs regulate the expression of the transcription factor $\mathrm{NF}-\kappa \mathrm{B}$, we also evaluated the inhibitory potential of the compounds on $\mathrm{NF}-\kappa \mathrm{B}$ activation. Only four chalcones, isoliquiritigenin (no. 10), butein (no. 12), homobutein (no. 15) and the glycoside marein (no. 21) showed HDAC inhibitory activity with $\mathrm{IC}_{50}$ values of $60-190 \mu \mathrm{M}$, whereas a number of compounds inhibited TNF $\alpha$-induced $N F-\kappa B$ activation with $\mathrm{IC}_{50}$ values in the range of 8-41 $\mu \mathrm{M}$. Interestingly, three chalcones (nos. 10, 12 and 15) inhibited both TNF $\alpha$-induced $\mathrm{NF}-\kappa \mathrm{B}$ activity and total HDAC activity of classes I, II and IV. Molecular modeling and docking studies were performed to shed light into dual activity and to draw structure-activity relationships among chalcones (nos. 1-21). To the best of our knowledge this is the first study that provides evidence for HDACs as potential drug targets for natural chalcones. The dual inhibitory potential of the selected chalcones on NF- $\kappa \mathrm{B}$ and HDACs was investigated for the first time. This study demonstrates that chalcones can serve as lead compounds in the development of dual inhibitors against both targets in the treatment of inflammation and cancer.
\end{abstract}

Correspondence to: Dr Marc Diederich, Laboratory of Molecular and Cellular Biology of Cancer, Cancer and Blood Research Foundation, Kirchberg Hospital, 9, rue Edward Steichen, L-2540 Luxembourg, Luxembourg

E-mail: marc.diederich@lbmcc.lu

Professor Deniz Tazdemir, ${ }^{4}$ Present address: School of Chemistry, National University of Ireland, Galway, University Road, Galway, Republic of Ireland

E-mail: deniz.tasdemir@nuigalway.ie

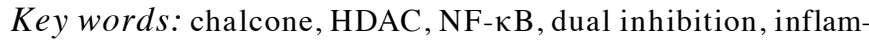
mation, cancer

\section{Introduction}

Acetylation is a pivotal post-transcriptional modification, which strongly influences chromatin structure and function (1). Due to its wide variety of targets, it is not only implicated in the regulation of gene expression via chromatin structure modifications but also in protein-protein interactions, protein stability, DNA binding, and subcellular localization (2). Histone acetylation is mediated by histone acetyltransferases (HATs). The resulting structural modification of chromatin leads to nucleosomal relaxation and altered transcriptional activation. The reverse reaction is mediated by histone deacetylases (HDACs), which induce deacetylation, chromatin condensation, and transcriptional repression (3). Alterations mediated by HAT/HDAC activities are not solely reduced to chromatin. Mounting evidence accentuates their involvement in lysine acetylation/deacetylation of non-histone substrates including transcription factors (such as NF- $\kappa \mathrm{B}, \mathrm{p} 53, \mathrm{GATA} 2, \mathrm{MEF} 2$ ) and chromatin-associated co-repressor proteins $(4,5)$. A balanced histone acetylation status is essential for the proper progress of cell proliferation, apoptosis and differentiation. An improper HDAC recruitment or activity, often leads to abnormal gene expression that is associated with cancer development (6), hence rendering HDAC as an excellent target in current cancer research. Two HDAC inhibitors, suberoylanilide hydroxamic acid (SAHA; vorinostat) and the natural product romidepsin (FK-228) are currently approved for cancer chemotherapy and many other inhibitors are in clinical trials (7). So far, eighteen human HDAC enzymes have been identified and grouped into four classes based on the structure of their accessory domains. Classes I, II, and IV, but not III, require a zinc molecule as an essential cofactor in their active site and are inhibited by $\mathrm{Zn}^{2+}$-binding HDAC inhibitors such as SAHA and the natural product, trichostatin A. Class I HDACs comprise HDACs 1, 2, 3 and 8, whereas class II HDACs include HDACs 4, 5, 6,7 and 9 that are larger in size than the other classes $(4,5)$. Recent publication of the X-ray crystal structure of HDAC8 (8) followed by several other HDACs (7) fuelled the research activity in the discovery and development of novel HDAC inhibitors.

The transcription factor $\mathrm{NF}-\kappa \mathrm{B}$ is a dimer of proteins belonging to the Rel family. It is an ubiquitous transcription factor present in all cell types. The most common form of $\mathrm{NF}-\kappa \mathrm{B}$ is the p65/p50 heterodimer. In most cells, NF- $\kappa \mathrm{B}$ 
complexes are localized to the cytosol as inactive forms with the inhibitor of $\kappa \mathrm{B}$ protein $(\mathrm{I} \kappa \mathrm{B})$. Phosphorylation of $\mathrm{I} \kappa \mathrm{B}$ results in its ubiquitination and subsequent proteasomemediated degradation. Activated NF- $\kappa \mathrm{B}$ then translocates to the nucleus where it transactivates more than 500 target genes (9). Many factors are known to activate NF- $\kappa \mathrm{B}$, including inflammatory cytokines such as tumor necrosis factor alpha $(\mathrm{TNF} \alpha)$ and interleukin (IL)-1, carcinogens (cigarette smoke), UV radiation, hyperglycemia and tumor promoters. Over the last decade, NF- $\kappa \mathrm{B}$ became a major target in drug discovery, due to its key role in cancer development, inflammation, cell proliferation and death (10).

Recent evidence indicates that the activation of various transcription factors, including NF- $\kappa \mathrm{B}$, is regulated through the interaction with HDAC proteins (11-13). Previous studies with various cell types showed that HDAC1 and HDAC2 negatively regulate NF- $\mathrm{KB}$ activity through direct interaction with the p65 (RelA) subunit of NF-kB $(14,15)$. Other studies suggest a physical interaction between p65 (RelA) and class I type HDACs (HDAC1, 2 and 3), where high expression of class I HDACs has been linked to increased nuclear translocation of p65 (RelA) (16). Remarkably, Class I HDAC isoforms are often overexpressed in various types of cancers where they are usually associated with a poor prognosis $(5,17,18)$. In addition, HDAC inhibitors are acknowledged as effective anti-inflammatory agents, some inhibiting NF-KB (19-21) and may therefore play an important role in the prevention of cancers that develop as a result of chronic inflammation. The combined antiproliferative as well as anti-inflammatory potency represents a highly attractive combination for the treatment of numerous chronic inflammatory conditions which are frequently associated with an increased risk of developing cancer.

Chalcones (1,3-diphenyl-2-propenones) are a group of aromatic compounds that represent a large class of natural products found in many medicinal plants, fruits, vegetables, spices and nuts. They are the natural precursors of flavonoids and display a variety of biological activities. Although the modes of action of this class of compounds are not fully understood, great efforts are devoted to elucidate the mechanisms underlying their promising anti-inflammatory and anticancer activities. Hence several natural chalcones have been reported to inhibit the NF- $\kappa \mathrm{B}$ signaling, and numerous synthetic derivatives have been evaluated in structure-activity relationship (SAR) studies $(22,23)$. Besides the NF- $\kappa \mathrm{B}$ inhibition, interference in microtubule formation is generally thought to be responsible for their anticancer activities $(24,25)$. Despite the cross-talk and modulation effects between NF- $\mathrm{KB}$ and HDACs, and structural similarity of chalcones to broadspectrum HDAC inhibitors SAHA and trichostatin A, HDACs have not been investigated as potential targets for natural chalcones. In this study, we aimed to test twenty-one commercially available chalcones (Table I) for dual HDACs and NF- $\kappa B$ inhibitory activities in vitro. Viability assays were also carried out to elucidate the cytotoxic potential of the chalcones against leukemia cells. We also aimed to explore SAR to determine the essential functionalities on the chalcone core for biological activity. We also performed molecular modeling and docking studies in an attempt to understand the potential mode and mode/site of binding of natural chalcones to NF- $\mathrm{KB}$ and class I type HDACs.

\section{Materials and methods}

Chalcones. Twenty-one natural chalcones, namely chalcone, 2'-hydroxychalcone, 2-hydroxychalcone, 4-hydroxychalcone, 4-methoxychalcone, 3,4-dimethoxychalcone, 4'-hydroxychalcone, 4'-methoxychalcone, 4,4'-dimethoxychalcone, isoliquiritigenin (2',4,4'-trihydroxychalcone), calomelanone (2',6'-dihydroxy-4,4'-dimethoxydihydrochalcone), butein (2',3-4,4'-tetrahydroxychalcone), flavokawain C (2',4-dihydroxy-4',6'-dimethoxychalcone), gymnogrammene ( $2^{\prime}, 6^{\prime}$-dihydroxy-4,4'-dimethoxychalcone), homobutein (2',4,4'-trihydroxy-3-methoxychalcone), 2,3-dimethoxy-2'-hydroxychalcone, flavokawain A (2'-hydroxy-4,4',6'-trimethoxychalcone), eriodictyolchalcone ( $2^{\prime}, 4^{\prime}, 6^{\prime}, 3,4-$ pentahydroxychalcone) phloretin $\left(2^{\prime}, 4,4 ', 6^{\prime}\right.$-tetrahydroxydihydrochalcone), phloridzin (phloretin-2'-O-glucoside) and marein (2',3,3',4,4'-pentahydroxy-4'-glucosylchalcone) were studied. Table 1 shows their chemical features and substitution patterns. Chalcone was purchased from Fluka (Steinheim, Germany), whereas all remaining chalcones were obtained from Extrasynthese (Genay Cedex, France) (purity $>97 \%$ ).

Cell culture and reagents. Human Philadelphia chromosomepositive chronic myelogenous leukemia cell line K562 was purchased from Deutsche Sammlung für Mikroorganismen und Zellkulturen (DSMZ, Braunschweig, Germany) and cultured in RPMI-1640 medium (Lonza, Verviers, Belgium) supplemented with $10 \%$ fetal calf serum (Hyclone, Perbio, Erembodegem, Belgium) and 1\% (v/v) antibiotic-antimycotic (Lonza, BioWhittaker ${ }^{\mathrm{TM}}$ ), at $37^{\circ} \mathrm{C}$, in a $5 \% \mathrm{CO}_{2}$, humidified atmosphere. Human recombinant TNF $\alpha$ (PeproTech, Rocky Hill, NJ, USA) was resuspended in $1 \mathrm{X}$ phosphate-buffered saline (PBS) sterile solution containing $0.5 \%$ bovine serum albumin (MP Biomedicals, Asse-Relegem, Belgium) to reach a final concentration of $10 \mu \mathrm{g} / \mathrm{ml}$.

HDAC activity/inhibition measurement. Direct HDAC inhibition by chalcone derivatives was estimated using K562 total extracts as an HDAC source and the enzymatic HDAC activity measurement was performed using a fluorometric HDAC assay kit (Active Motif, Rixensart, Belgium) according to the manufacturer's instructions. Briefly, after being washed twice with ice-cold 1X PBS, cells were pelleted by centrifugation, and lysed in $\mathrm{M}-\mathrm{PER}^{\circledast}$ mammalian protein extraction reagent (Pierce, Erembodegem, Belgium) and supplemented with 1X protease inhibitor cocktail (Roche, Prophac, Luxembourg). The cell suspension was gently mixed on an orbital shaker for $15 \mathrm{~min}$ and centrifuged at $14,000 \mathrm{x}$ g at $4^{\circ} \mathrm{C}$ for $15 \mathrm{~min}$. Protein content was assessed using the Bradford assay (BioRad, Nazareth, Belgium), and $10 \mu \mathrm{g}$ of proteins were incubated with vehicle or various concentrations of the different chalcones for $1 \mathrm{~h}$ at $37^{\circ} \mathrm{C}$ in the presence of an HDAC fluorometric substrate. Subsequently, the HDAC assay developing solution was added and after $15 \mathrm{~min}$ of incubation at room temperature, the fluorescence was measured using a Gemini EM microplate spectrofluorometer (Molecular Devices, Belgium) with excitation at $360 \mathrm{~nm}$ and emission at $460 \mathrm{~nm}$. The measured activities were normalized by the vehicle-treated control enzyme activities and $\mathrm{IC}_{50}$ values were calculated. 


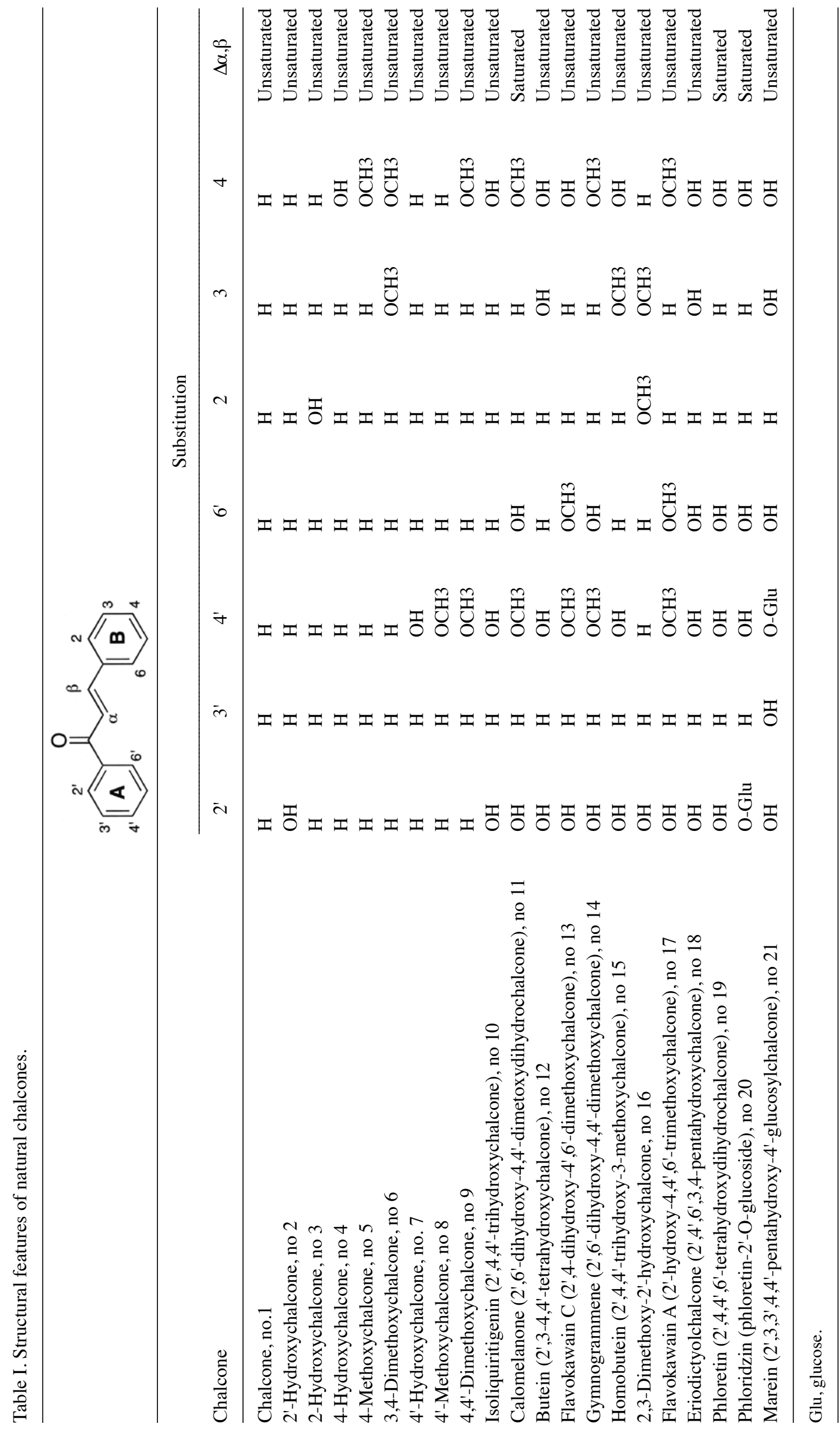


A
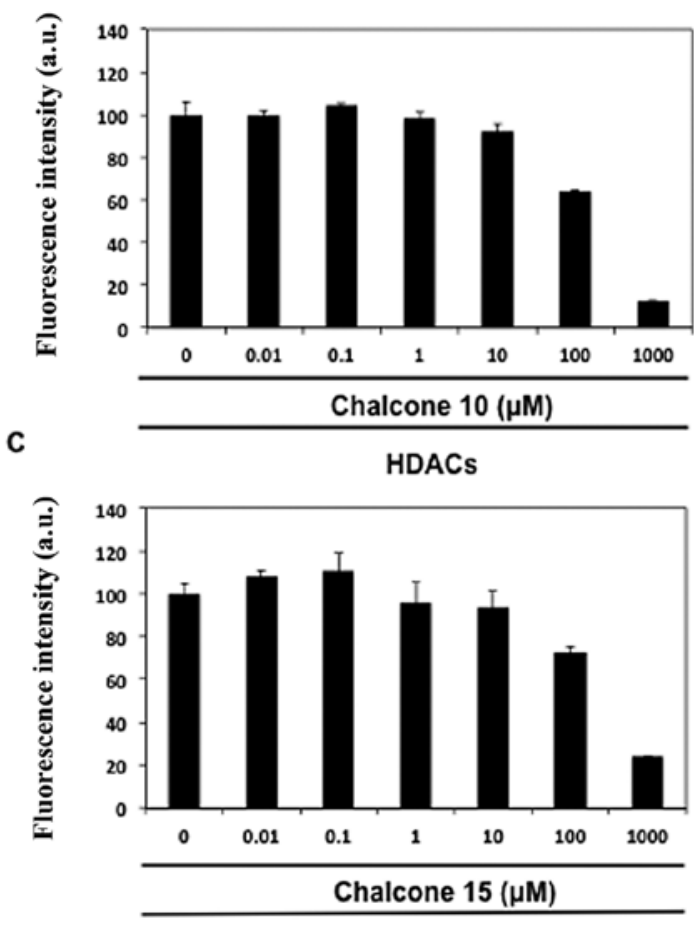

HDACs
B

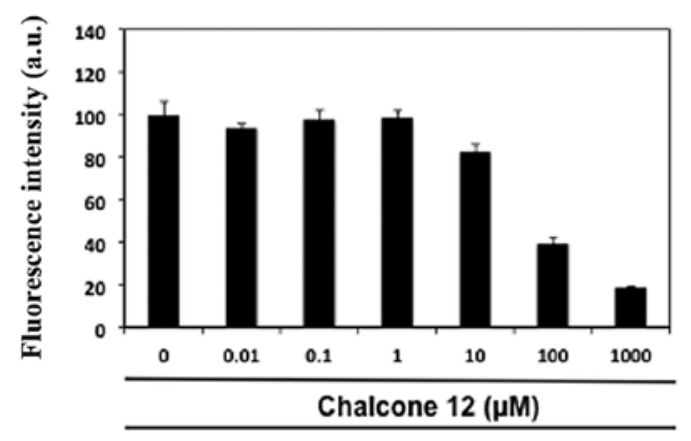

D

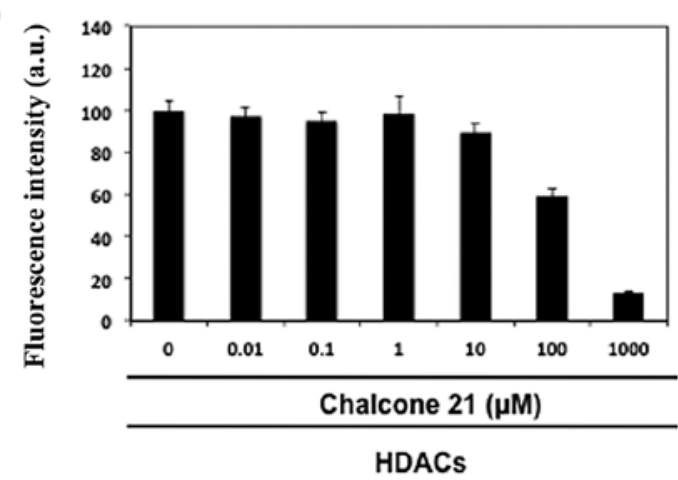

Figure 1. Inhibition of HDAC activity by active chalcone derivatives. Total protein extracts from K562 cells were incubated with vehicle (0) or various concentrations of the (A) chalcone 10 (B) chalcone 12 (C) chalcone 15 or (D) chalcone 21 for $1 \mathrm{~h}$ in the presence of an HDAC fluorometric substrate. Fluorescence was measured using a Gemini EM microplate spectrofluorometer and normalized by the vehicle-treated control enzyme activities. Vehicle-treated control corresponds to 0 on the chart. Results are presented as a mean \pm SD of at least three independent experiments.

Transient transfections. Transient transfections of K562 cells were performed as previously described (26).

In vitro cytotoxicity assays (viability assay). The in vitro growth inhibitory values of chalcone derivatives on the K562 cell line were determined as detailed previously (26).

Molecular modeling and docking studies. The 2D structures of chalcone molecules were drawn using SketchEI and transferred into the VEGA ZZ molecular modeling software $(27,28)$ to generate $3 \mathrm{D}$ structures. All molecules were saved into a single mol file, that was used as input for the OMEGA, OpenEye Scientific Software (Omega version 2.3.2; http:// www.openeye.com) to generate a maximum of 2 low energy conformers with default values. These conformations were stored as OEB file extension format and their 3D similarity was compared using the Rocs, OpenEye Scientific Software (Rocs version 2.3.1; http://www.openeye.com). E-Dragon Software (29) was utilized to calculate constitutional and molecular property descriptors. The descriptors selected to describe the SAR were selected using Partial Least Squares regression as implemented in the PLSR module of Virtual Computational Chemistry Laboratory (29) and Gretl software was used to calculate the correlation between the logarithm of the activity and predicted molecular properties.

The molecular docking was carried out using Glide software (Grid-Based Ligand Docking With Energetics) (Schrodinger Inc., Portland, OR, USA) $(30,31)$ after the docking targets were prepared using Protein Preparation Wizard workflow in Maestro (Schrodinger Inc.) by removing water molecules, adding the hydrogen atoms and assigning all atom force field (OPSL-2005) charges and atom types. The position of all atoms was adjusted by minimizations until the average root mean square deviation reached $0.3 \AA$. The crystal structures of HDAC8 wild-type and variant D101 complexed with ligands [Protein Data Bank (pdb) entries 1T69 and 3EZT] were used for molecular docking of chalcones into the protein active site. The box encompassing the active site was selected based on the position of co-crystalized ligands complex as described in a previous study (32). The crystal structure of NF- $\kappa \mathrm{B}$ complexed to DNA was chosen as a target system to elucidate binding modes of chalcones (pdb entry 1NFK). Prior to docking the DNA molecule was removed and the coordinates of the enclosing box of $30 \AA$ (center at $\mathrm{x}=-1,1958 \AA$; $\mathrm{y}=9.0149 \AA$; $\mathrm{z}=$ $19,7598 \AA$ ) were encompassing the active site residues involved in hydrogen bonds with the $\mathrm{NF}-\kappa \mathrm{B}$ recognition site of DNA (Arg54, Arg56, Tyr57, Cys59, Lys241, Gln306 and Thr143) (35). Flexible ligand docking simulations were carried out with Glide using the default settings. The ten best poses obtained using the Extra-Precision Glide (Glide XP) mode were selected for analysis. The most favorable poses of molecules showing activity were subjected to further energy minimization using Macromodel 9.1 and OPLS2005 force field.

\section{Results}

Inhibition of HDAC activity by chalcone derivatives. The effect of chalcone derivatives (nos. 1-21) was examined on total 
A

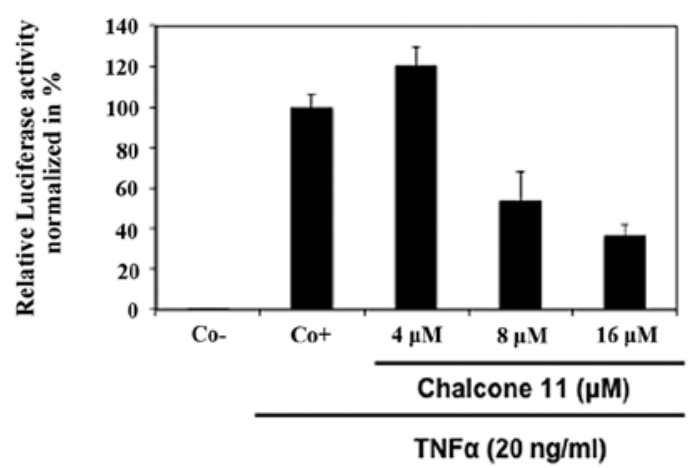

B

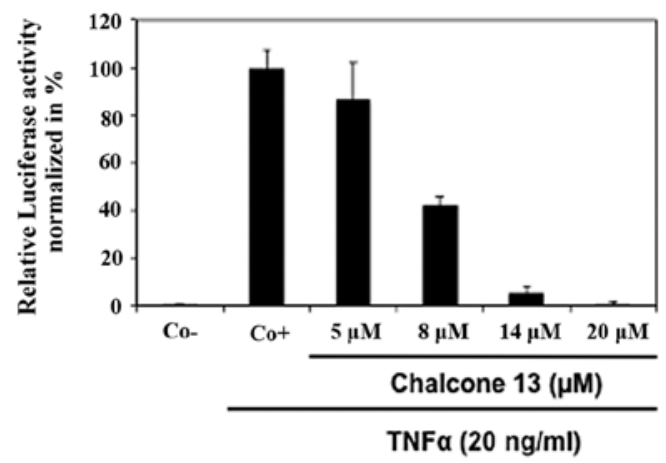

Figure 2. Inhibition of TNF $\alpha$-induced NF- $\kappa \mathrm{B}$ activation by (A) chalcone 11 and (B) chalcone 13 . K562 cells were transiently transfected with firefly luciferase vector (NF- $\mathrm{B}$ pGL4), and ph-RG-tk Renilla plasmid for $24 \mathrm{~h}$. After transfection, K562 cells were treated with chalcone derivatives at the different concentrations for $2 \mathrm{~h}$ followed by a TNF $\alpha$-treatment $(20 \mathrm{ng} / \mathrm{ml})$ during $6 \mathrm{~h}$. Results are expressed as a ratio of the measured luminescence of the firefly luciferase vector and the luminescence of Renilla plasmid. Results are presented as a mean \pm SD of three independent experiments. Negative control (Co-) corresponds to DMSO treated cells, without TNF $\alpha$ activation, positive control $(\mathrm{Co}+)$ corresponds to DMSO treated cells activated by TNF $\alpha$.

HDAC activity using a fluorescence HDAC assay. As shown in Table II, four chalcone aglycones, namely isoliquiritigenin (no. 10), butein (no. 12), homobutein (no. 15) and the glycoside marein (no. 21), reduced HDAC activity in a concentrationdependent manner $\left(\mathrm{IC}_{50}\right.$ values 60-190 $\mu \mathrm{M}$, Fig. 1). Butein (no. 12) appeared to be the best inhibitor of HDAC activity. Other chalcone derivatives were assumed as inactive, because they were unable to provide distinct inhibitory effect even at the highest test concentration $(1000 \mu \mathrm{M})$.

Inhibition of $T N F \alpha$-induced $N F-\kappa B$ activation by chalcones. By using a luciferase-based in cellulo $\mathrm{NF}-\kappa \mathrm{B}$ reporter assay, chalcones were evaluated for $\mathrm{TNF} \alpha$-induced $\mathrm{NF}-\kappa \mathrm{B}$ transcription inhibition activity (Table II). Flavokawain $\mathrm{C}$ (no. 13) was the most potent NF- $\mathrm{BB}$ inhibitor, followed by the dihydrochalcone calomelanone (no. 11) with $\mathrm{IC}_{50}$ values of 8 and $11 \mu \mathrm{M}$, respectively (Fig. 2). Chalcones 4-hydroxychalcone (no. 4), 4-methoxychalcone (no. 5), 4'-hydroxychalcone (no. 7), isoliquiritigenin (no. 10), butein (no. 12), homobutein (no. 15) and phloretin (no. 19) also demonstrated good potential with $\mathrm{IC}_{50}$ values ranging between $24-41 \mu \mathrm{M}$. Three polymethoxychalcones, i.e. 4,4'-dimethoxychalcone (no. 9), gymnogrammene (no. 14), flavokawain A (no. 17), as well as cone eriodictyolchalcone (no. 18) and two chalcone glycosides, phloridzin (no. 20) and marein (no. 21), which showed no or limited inhibitory activity at $200 \mu \mathrm{M}$ concentration, were considered as inactive. The remaining compounds, chalcone (no. 1), 2'-hydroxychalcone (no. 2), 2-hydroxychalcone (no. 3), 3,4-dimethoxychalcone (no. 6), 4'-methoxychalcone (no. 8) and 2,3-dimethoxy-2'-hydroxychalcone (16) were cytotoxic at concentrations, which inhibited NF- $\mathrm{KB}$ activation.

Docking studies of chalcones 12 and chalcone 21 within the active site of $H D A C 8$. In order to shed light on the potential mode of action of chalcones, and to understand why some chalcones inhibit either NF- $\mathrm{B}$ or HDACs and some inhibit both, we have carried out molecular modeling, molecular similarity and docking studies. The compounds were docked into the binding sites of HDAC8, and the best studied HDAC enzyme was selected based on the position of the co-crystal-
Table II. Biological activity of natural chalcones.

\begin{tabular}{|c|c|c|c|}
\hline Chalcone & $\begin{array}{c}\text { HDAC } \\
\text { inhibition, } \\
\mathrm{IC}_{50}(\mu \mathrm{M})\end{array}$ & $\begin{array}{c}\mathrm{NF}-\kappa \mathrm{B} \\
\text { inhibition, } \\
\mathrm{IC}_{50}(\mu \mathrm{M})\end{array}$ & Viability \\
\hline 1 & $>1000$ & n.m. & 14 \\
\hline 2 & $>1000$ & n.m. & 28 \\
\hline 3 & $>1000$ & n.m. & 2 \\
\hline 4 & $>1000$ & 24 & 31 \\
\hline 5 & $>1000$ & 29 & 35 \\
\hline 6 & $>1000$ & n.m. & 38 \\
\hline 7 & $>1000$ & 28 & 16 \\
\hline 8 & $>1000$ & n.m. & 38 \\
\hline 9 & $>1000$ & $>200$ & n.t. \\
\hline 10 & 110 & 32 & 44 \\
\hline 11 & $>1000$ & 11 & 31 \\
\hline 12 & 60 & 38 & 13 \\
\hline 13 & $>1000$ & 8 & 13 \\
\hline 14 & $>1000$ & $>200$ & n.t. \\
\hline 15 & 190 & 38 & 29 \\
\hline 16 & $>1000$ & n.m. & 12 \\
\hline 17 & $>1000$ & $>200$ & n.t. \\
\hline 18 & $>1000$ & $>200$ & n.t. \\
\hline 19 & $>1000$ & 41 & 59 \\
\hline 20 & $>1000$ & $>200$ & n.t. \\
\hline 21 & 100 & $>200$ & n.t. \\
\hline Standards & $0.14^{\mathrm{b}}$ & $6.0^{\mathrm{a}}$ & $1.4^{\mathrm{a}}$ \\
\hline
\end{tabular}

n.m., not measurable due to toxicity after transfection; n.t., not tested

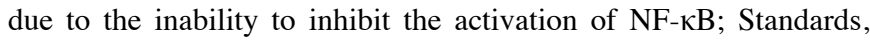
${ }^{\text {aheteronemin, }}{ }^{\mathrm{b}}$ suberoylanilide hydroxamic acid.

ized ligand in the crystal structure of the complex (pdb entries 1T69 and 3ETZ) (32). The GlideScore values were compared to the activities that were experimentally obtained (Table III). 
Table III. GlideScore values obtained for HDAC and NF-кB proteins.

\begin{tabular}{cccc}
\hline Chalcone & $\begin{array}{c}\text { Docking scores } \\
\text { against 1T69 }\end{array}$ & $\begin{array}{c}\text { Docking scores } \\
\text { against 3ETZ }\end{array}$ & $\begin{array}{c}\text { Docking scores } \\
\text { against 1NFK }\end{array}$ \\
\hline & & & \\
1 & -6.83 & -5.27 & -2.39 \\
2 & -5.90 & -6.08 & -4.05 \\
3 & -5.40 & -6.35 & -3.06 \\
4 & -4.71 & -6.27 & -2.38 \\
5 & -5.52 & -5.34 & -3.22 \\
6 & -4.92 & -5.87 & -2.76 \\
7 & -4.88 & -5.96 & -2.88 \\
8 & -5.78 & -5.86 & -2.48 \\
9 & -5.88 & -5.70 & -2.6 \\
10 & -6.03 & -7.03 & -2.84 \\
11 & -6.75 & -7.21 & -3.15 \\
12 & -7.00 & -8.00 & -4.71 \\
13 & -5.32 & -7.45 & -3.48 \\
14 & -5.63 & -6.52 & -4.29 \\
15 & -6.44 & -7.06 & -0.71 \\
16 & -5.31 & -6.57 & -3.1 \\
17 & -4.47 & -6.79 & -1.75 \\
18 & -5.69 & -7.24 & -4.06 \\
19 & -5.15 & -6.47 & 0.12 \\
20 & -6.85 & -7.57 & -5.53 \\
21 & -8.08 & -10.51 & -6.03 \\
\hline
\end{tabular}

The results of the docking indicated that all chalcones could favorably bind in the active site, although not all molecules showed activity. The most active molecule 12 had a less favorable GlideScore than chalcone 21 that exhibited the most favorable binding. The binding mode of these two molecules is different (Fig. 3) which may be the result of the large active site of HDAC8 which accommodates two (4-(dimethylamino)$\mathrm{N}$-[7-(hydroxyamino)-7-oxoheptyl]benzamide) moieties in the interior pocket of the protein. Furthermore, the docking could not distinguish the third active molecule 10 from the rest of the group. There is a clear difference between the GlideScore of the binding of 21 to 20 . However, 20 binds almost as good as 12 and better than 10, resulting in the absence of the correlation between activity and binding affinity determined by Glide. We hypothesize that molecules that are not active could possibly bind preferably elsewhere on the protein surface rather than on the active site.

The docking studies of chalcones were also carried out using a crystal structure of NF- $\mathrm{KB}$ dimer in complex with duplex DNA. We followed a procedure reported by Piccagli et al (33) and have chosen the DNA recognition surface to define the docking target. The binding site included residues Arg54, Arg56, Tyr57, Cys59, Lys241, Gln306 and Thr143 to gain information on the interaction of our compounds with NF- $\kappa B$. As shown in Table III, there is a lack of correlation between the activity and the GlideScore results. This can be due to non-specific binding. Moreover, chalcones could act

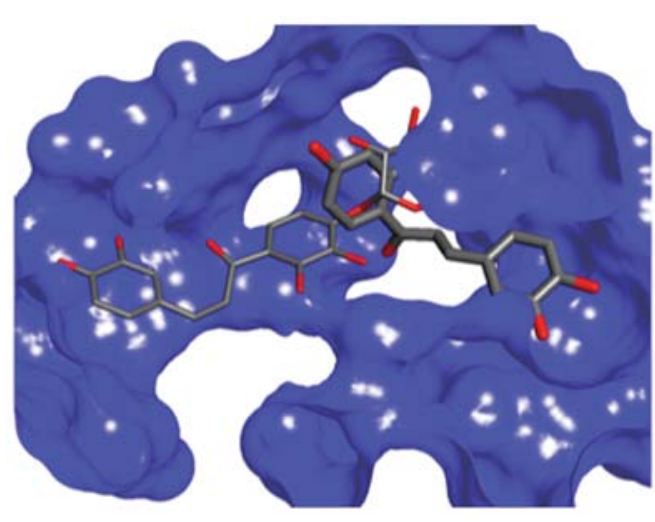

Figure 3. Best binding poses of chalcone 12 (thick stick representation) and chalcone 21 (thin stick representation) within the active site of the HDAC8 represented by surface only. The docking was carried out using Glide and DS Visualizer 3.1 was used to prepare the image.

on a different active site of NF- $\mathrm{kB}$. To further rationalize the activity of this class of compounds we have elucidated the SARs and predicted more than 1600 molecular properties for all molecules using EDragon software (29). This analysis was not aimed to lead to the development of predictive models since the data set is small (fifteen molecules with measured NF- $\kappa \mathrm{B}$ activity) and thus we did not create training and test sets. Two different sets of molecules were subjected to partial least square regression using PLSR module of the Virtual Computational Chemistry Laboratory to determine which constitutional and molecular properties correlated with the negative logarithm of activity (pNF- $\mathrm{kB}$ ). The first group consisted of all fifteen tested molecules and the observed correlation was not satisfactory $\left(r^{2}=0.53\right)$, leading us to examine the second smaller group, consisting of only nine molecules that displayed activity. The PLS results indicated that a combination of nineteen descriptors correlated with activity $\left(r^{2}=0.99\right)$. There were some highly correlated and irrelevant descriptors selected to describe correlation. The selection of descriptors was optimized by developing least square methods using the Gretl software and removing descriptors until a minimal number of descriptors was obtained with a good correlation $\left(r^{2}=0.914, s=0.250, n=9, F=3.48\right)$. The formula used was the following: pNF- $\kappa \mathrm{B}=-79.78( \pm 48.55)-0.425( \pm .0 .344) * \mathrm{SS}+$ $31.582( \pm 24 .)^{*} \mathrm{Mp}+43.847( \pm 25.978)^{*} \mathrm{ARR}+1.868( \pm 1.619)^{*} \mathrm{Hy}$ $+0.265( \pm .468)^{*} \mathrm{MLOGP}+2.793( \pm 1.682)^{*} \mathrm{nBO}$.

The list and values of descriptors, observed and calculated are shown in Table IV. The statistics indicate reasonable descriptive value of the model that shows which molecular properties influence activity of the molecules in the NF- $\mathrm{KB}$ assay. Since we could not develop a satisfactory model that would differentiate the active and inactive molecules, we examined the molecular similarity and differences between most active molecules and the inactive ones. Conformational search carried out by Omega software and the Merck Molecular Force Field force revealed that all molecules can exist in several different conformations due to the free rotation around bonds between carbonyl carbon and neighboring groups. ROCS search and comparison of electrostatic forces showed that unsurprisingly molecules are similar (Shape Tanimoto coefficients are between 0.94 and 0.0 .69 and Tverstsky coefficients are between 0.91 and 0.71 ). The highest similarity was 
Table IV. Predicted molecular properties correlating to the activity of potent chalcones in the NF- $\kappa \mathrm{B}$ assay.

\begin{tabular}{|c|c|c|c|c|c|c|c|c|}
\hline Chalcone & Ss & $\mathrm{Mp}$ & $\mathrm{nBO}$ & Hy & MLOGP & ARR & $\begin{array}{c}\text { Observed } \\
\text { pNF-кB }\end{array}$ & $\begin{array}{c}\text { Calculated } \\
\text { pNF- } \kappa B\end{array}$ \\
\hline 4 & 41.67 & 0.71 & 18 & -0.371 & 3.148 & 0.667 & 4.62 & 4.59 \\
\hline 5 & 41.17 & 0.69 & 19 & -0.873 & 3.398 & 0.632 & 4.54 & 4.56 \\
\hline 7 & 41.67 & 0.71 & 18 & -0.371 & 3.148 & 0.667 & 4.55 & 4.59 \\
\hline 10 & 53.00 & 0.69 & 20 & 1.096 & 2.552 & 0.600 & 4.49 & 4.37 \\
\hline 11 & 56.67 & 0.65 & 23 & 0.304 & 2.334 & 0.522 & 4.96 & 4.97 \\
\hline 12 & 58.67 & 0.68 & 21 & 1.928 & 1.764 & 0.571 & 4.42 & 4.51 \\
\hline 13 & 57.67 & 0.67 & 23 & 0.304 & 1.746 & 0.522 & 5.10 & 5.02 \\
\hline 15 & 58.17 & 0.67 & 22 & 0.545 & 1.062 & 2.013 & 4.42 & 4.51 \\
\hline 19 & 58.67 & 0.68 & 21 & 1.928 & 1.764 & 0.571 & 4.39 & 4.40 \\
\hline
\end{tabular}

Ss, Sum of Kier-Hall electrotopological states; Mp, mean atomic polarizability (scaled on Carbon atom); nBO, number of non-H bonds; Hy, hydrophilic factor; MLOGP, Moriguchi octanol-water partition coefficient $(\log \mathrm{P})$; $\mathrm{ARR}$, aromatic ratio; $\mathrm{pNF}-\mathrm{\kappa B}$, negative logarithm values of activity observed in NF- $\mathrm{B}$ assay.

observed between the most active chalcone molecule 13 and inactive 9, indicating that shape and electrostatic properties are not sufficient to explain the different activities of the group.

\section{Discussion}

Chronic inflammation has been linked to most incurable illnesses, including cancer, cardiovascular and neurodegenerative diseases. Cancer is regulated by a large number of genes that are modulated by transcription factors, such as $\mathrm{NF}-\kappa \mathrm{B}$, which controls genes involved in inflammation, proliferation, angiogenesis and metastasis (23). Any disturbance in the corresponding pathways leads to the activation of NF- $\kappa \mathrm{B}$ and release of cytokines, thus contributing to the initiation and progression of tumorigenesis. On the other hand, acetylation and deacetylation act as regulating mechanisms for activation or inactivation of various transcription factors, including NF- $\mathrm{kB}$. This process is mediated by HDAC and can consequently be modulated by HDAC inhibitors (34). Protein complexes involved in the regulation of cell-cycle progression and apoptosis are also controlled by this mechanism (2). The reversible acetylation appears to regulate the interaction between $\mathrm{p} 65$ and $\mathrm{I \kappa B}$, and controls the duration of the NF- $\mathrm{KB}$ response. NF- $\mathrm{KB}$ activation leads to apoptosis resistance. Several NF- $\kappa \mathrm{B}$ inhibitors showed potential to overcome this resistance and induce apoptosis $(35,36)$. Thus, inhibition of NF- $\kappa \mathrm{B}$ may sensitize cancer cells and eventually lead to the induction of apoptosis. Interestingly, in our study, chalcones $4,5,7,10,11,12,13,15$ and 19 inhibited both NF- $\kappa B$ and the viability of K562 cells.

Interestingly, three chalcones (nos. 10, 12, and 15) inhibited both NF- $\kappa$ B and HDAC activity. Nevertheless, underlying mechanisms in the action of chalcones as dual inhibitors remain to be elucidated in the future. To our knowledge, the mechanisms that link both inhibitory activities were first reported herein. As an example, upon interaction with histone deacetylase 3 (HDAC3), p65 is deacetylated leading to efficient interaction with IKB and subsequent activation via the canonical pathway (37). Compounds that efficiently inhibit
HDAC 3 and other HDAC isoforms are considered interesting NF- $\mathrm{BB}$ inhibitors. Moreover, transcription factor STAT1 is physiologically acetylated and binds p65, thus inhibiting NF- $\kappa$ B. STAT1-associated HDAC were described to deacetylate STAT1 leading to the liberation of p65 and subsequent activation of the canonical NF- $\kappa B$ pathway. Inhibitors of HDAC activity contribute to a shift towards acetylated STAT1 actively interacting with NF- $\mathrm{KB}$ and thus inhibiting activation of p65 required for inflammatory cell signaling (38). Even though the inhibitory activity of selected chalcones appears weak when only the total HDAC activity is assessed, inhibitory effect against specific HDAC isoforms are generally stronger as shown for tubastatin A (39).

Attempts to generate HDAC inhibitors generally focus on varying the cap group to exploit variability in the HDAC surface surrounding the active site. However, although efforts are being made to identify truly class- or isoform-selective HDAC inhibitors with anticancer and anti-inflammatory properties, the current list of such compounds remains relatively poor. One of the main reasons is the lack of structural determinants of selective HDAC inhibition due to the challenge of studying the interaction of small inhibitory molecules with multiple large protein complexes that encompass HDAC activities, which are often dependent on or regulated by these complexes. Accordingly, the selectivity of small inhibitory molecules may depend on the context of HDAC complexes and requires investigation (40). The small size of the library limits the ability to obtain common structural features necessary for individual or dual target activity.

A number of SAR studies have been performed on synthetic chalcones and their biological effects, including NF- $\mathrm{\kappa B}$ inhibition $(22,23)$. To our knowledge this is the first study looking at potential SARs among natural chalcones. In the present study, some chalcones showed interesting NF- $\kappa \mathrm{B}$ inhibitory potential, and some empirical SARs have been obtained. Our results show that chalcone, the parent compound, or its 2'-hydroxy- or 2-hydroxy- derivatives (nos. 1-3) did not express any NF- $\kappa \mathrm{B}$ inhibition potential. We were able to draw some SARs originating from the substitutions with an electron donating 
functional group such as methoxy or hydroxy, at positions 4, 4' and 6'. Chalcone glycosides (nos. 20 and 21) were not active, which might be due to the reduced permeability through cell membranes. In addition, all chalcones with reported NF- $\kappa \mathrm{B}$ activity contain a highly electrophilic $\alpha, \beta$-unsaturated carbonyl moiety and calomelanone and phloretin are the first examples of dihydrochalcones with such activity. For HDAC activity clearer trends were observed, i.e. hydroxy groups at C-2', C-4', C-3 and C-4 were essential. This substitution pattern also appears to be important for dual activity. The empirical SARs prompted us to perform some molecular modeling-docking studies on both targets. The molecular modeling investigations could not provide a definite rationale for dual activity of chalcones and failed to provide criteria for distinguishing the active molecules from inactive. There could be many reasons underlying this observation. Most of the molecules have low molecular weight (between 200 to $275 \mathrm{Da}$ ) and as such can be considered as fragments. This could lead to non-specific binding and lack of correlation between molecular properties and activity.

Some of the compounds tested herein have previously been reported as $\mathrm{NF}-\kappa \mathrm{B}$ inhibitors including isoliquiritigenin (no. 10) (41) and butein (no. 12) (42). We have previously demonstrated that 4'-hydroxychalcone showed $26 \mathrm{~S}$ protease inhibition activity on three different proteolytic activities (chymotrypsin, trypsin- and caspase-like) in a dose-dependent manner (26). The involvement of natural chalcones in cancer tumorigenesis has been reviewed (43). To the best of our knowledge this is the first study aiming to screen a natural chalcone library and attempting to draw SARs among them. Several natural chalcones emerged as relatively good inhibitors of NF- $\kappa \mathrm{B}$. Additionally, HDAC was identified as a novel potential target for the chalcones described.

\section{Acknowledgements}

During this project B.O. and M.S. were supported by Télévie grants. In addition, M.S. was supported by a 'Waxweiler grant for cancer prevention research' from the Action Lions 'Vaincre le Cancer'. This work was supported by the 'Recherche Cancer et Sang' foundation, 'Recherches Scientifiques Luxembourg' association and the Télévie Luxembourg. The authors thank 'Een Häerz fir Kriibskrank Kanner' association and the Action Lions 'Vaincre le Cancer' for generous support. Publication costs were covered by the Fonds National de la Recherche Luxembourg.

\section{References}

1. Grunstein M: Histone acetylation in chromatin structure and transcription. Nature 389: 349-352, 1997.

2. Minucci S and Pelicci PG: Histone deacetylase inhibitors and the promise of epigenetic (and more) treatments for cancer. Nat Rev Cancer 6: 38-51, 2006.

3. ten Holte P, Van Emelen K, Janicot M, Fong PC, de Bono JS and Arts J: HDAC inhibition in cancer therapy: an increasingly intriguing tale of chemistry, biology and clinical benefit. In: Cancer. Topics in Medicinal Chemistry. Bradbury RH (ed). Springer Verlag, Berlin, pp293-332, 2007.

4. Folmer F, Orlikova B, Schneckenburger M, Dicato M and Diederich M: Naturally occurring regulators of histone acetylation/deacetylation. Curr Nutr Food Sci 6: 78-99, 2010.

5. Seidel C, Schnekenburger M, Dicato M and Diederich M: Histone deacetylase modulators provided by Mother Nature. Genes Nutr: Feb. 12, 2012 (Epub ahead of print)
6. Gibbons RJ: Histone modifying and chromatin remodelling enzymes in cancer and dysplastic syndromes. Hum Mol Genet 14 (Suppl 1): R85-R92, 2005.

7. Lombardi PM, Cole KE, Dowling DP and Christianson DW: Structure, mechanism, and inhibition of histone deacetylases and related metalloenzymes. Curr Opin Struct Biol 21: 735-743, 2011.

8. Vannini A, Volpari C, Filocamo G, et al: Crystal structure of a eukaryotic zinc-dependent histone deacetylase, human HDAC8, complexed with a hydroxamic acid inhibitor. Proc Natl Acad Sci USA 101: 15064-15069, 2004.

9. Gupta SC, Sundaram C, Reuter S and Aggarwal BB: Inhibiting NF-kappaB activation by small molecules as a therapeutic strategy. Biochim Biophys Acta 1799: 775-787, 2010.

10. Vallabhapurapu S and Karin M: Regulation and function of NF-kappaB transcription factors in the immune system. Annu Rev Immunol 27: 693-733, 2009.

11. Batra S, Sahu RP, Kandala PK and Srivastava SK: Benzyl isothiocyanate-mediated inhibition of histone deacetylase leads to NF-kappaB turnoff in human pancreatic carcinoma cells. Mol Cancer Ther 9: 1596-1608, 2010.

12. Jung ID, Lee JS, Jeong YI, et al: Apicidin, the histone deacetylase inhibitor, suppresses Th1 polarization of murine bone marrow-derived dendritic cells. Int J Immunopathol Pharmacol 22: 501-515, 2009.

13. Roth SY, Denu JM and Allis CD: Histone acetyltransferases. Annu Rev Biochem 70: 81-120, 2001.

14. Kiernan R, Bres V, Ng RW, et al: Post-activation turn-off of NF-kappa B-dependent transcription is regulated by acetylation of p65. J Biol Chem 278: 2758-2766, 2003.

15. Hu J and Colburn NH: Histone deacetylase inhibition down-regulates cyclin D1 transcription by inhibiting nuclear factor-kappaB/ p65 DNA binding. Mol Cancer Res 3: 100-109, 2005.

16. Lehmann A, Denkert C, Budczies J, et al: High class I HDAC activity and expression are associated with RelA/p65 activation in pancreatic cancer in vitro and in vivo. BMC Cancer 9: 395, 2009.

17. Florean C, Schnekenburger M, Grandjenette C, Dicato M and Diederich M: Epigenomics of leukemia: from mechanisms to therapeutic applications. Epigenomics 3: 581-609, 2011.

18. Schnekenburger $M$ and Diederich $M$ : Epigenetics offer new horizons for colorectal cancer prevention. Curr Colorectal Cancer Rep 8: 66-81, 2012.

19. Papeleu P, Wullaert A, Elaut G, et al: Inhibition of NF-kappaB activation by the histone deacetylase inhibitor 4-Me2N-BAVAH induces an early G1 cell cycle arrest in primary hepatocytes. Cell Prolif 40: 640-655, 2007.

20. Fabre C, Grosjean J, Tailler M, et al: A novel effect of DNA methyltransferase and histone deacetylase inhibitors: NFkappaB inhibition in malignant myeloblasts. Cell Cycle 7: 2139-2145, 2008.

21. Halili MA, Andrews MR, Sweet MJ and Fairlie DP: Histone deacetylase inhibitors in inflammatory disease. Curr Top Med Chem 9: 309-319, 2009.

22. Srinivasan B, Johnson TE, Lad R and Xing C: Structure-activity relationship studies of chalcone leading to 3-hydroxy-4,3',4',5'tetramethoxychalcone and its analogues as potent nuclear factor kappaB inhibitors and their anticancer activities. J Med Chem 52: 7228-7235, 2009.

23. Yadav VR, Prasad S, Sung B and Aggarwal BB: The role of chalcones in suppression of NF-kappaB-mediated inflammation and cancer. Int Immunopharmacol 11: 295-309, 2011.

24. Edwards ML, Stemerick DM and Sunkara PS: Chalcones: a new class of antimitotic agents. J Med Chem 33: 1948-1954, 1990.

25. Lawrence NJ, McGown AT, Ducki S and Hadfield JA: The interaction of chalcones with tubulin. Anticancer Drug Des 15: 135-141, 2000.

26. Orlikova B, Tasdemir D, Golais F, Dicato M and Diederich M: The aromatic ketone 4'-hydroxychalcone inhibits TNFalphainduced NF-kappaB activation via proteasome inhibition. Biochem Pharmacol 82: 620-631, 2011.

27. Pedretti A, Villa L and Vistoli G: VEGA - an open platform to develop chemo-bio-informatics applications, using plug-in architecture and script programming. J Comput Aided Mol Des 18: 167-173, 2004.

28. Pedretti A, Villa, L and Vistoli G: Atom-type description language: a universal language to recognize atom types implemented in the VEGA program. Theor Chem Acc 109: 229-232, 2003.

29. Tetko IV, Gasteiger J, Todeschini R, et al: Virtual computational chemistry laboratory - design and description. J Comput Aided Mol Des 19: 453-463, 2005. 
30. Friesner RA, Banks JL, Murphy RB, et al: Glide: a new approach for rapid, accurate docking and scoring. 1. Method and assessment of docking accuracy. J Med Chem 47: 1739-1749, 2004.

31. Halgren TA, Murphy RB, Friesner RA, et al: Glide: a new approach for rapid, accurate docking and scoring. 2. Enrichment factors in database screening. J Med Chem 47: 1750-1759, 2004

32. Ortore G, Di Colo F and Martinelli A: Docking of hydroxamic acids into HDAC1 and HDAC8: a rationalization of activity trends and selectivities. J Chem Inf Model 49: 2774-2785, 2009.

33. Piccagli L, Fabbri E, Borgatti M, et al: Docking of molecules identified in bioactive medicinal plants extracts into the p50 NF-kappaB transcription factor: correlation with inhibition of NF-kappaB/DNA interactions and inhibitory effects on IL-8 gene expression. BMC Struct Biol 8: 38, 2008.

34. Di Gennaro E, Bruzzese F, Caraglia M, Abruzzese A and Budillon A: Acetylation of proteins as novel target for antitumor therapy: review article. Amino Acids 26: 435-441, 2004.

35. Shen KH, Chang JK, Hsu YL and Kuo PL: Chalcone arrests cell cycle progression and induces apoptosis through induction of mitochondrial pathway and inhibition of nuclear factor kappa $B$ signalling in human bladder cancer cells. Basic Clin Pharmacol Toxicol 101: 254-261, 2007.

36. Lee ST, Wong PF, Cheah SC and Mustafa MR: Alpha-tomatine induces apoptosis and inhibits nuclear factor-kappa $\mathrm{B}$ activation on human prostatic adenocarcinoma PC-3 cells. PLoS One 6 : e18915, 2011.
37. Chen L, Fischle W, Verdin E and Greene WC: Duration of nuclear NF-kappaB action regulated by reversible acetylation. Science 293: 1653-1657, 2001.

38. Krämer OH, Baus D, Knauer SK, et al: Acetylation of Stat1 modulates NF-kappaB activity. Genes Dev 20: 473-485, 2006.

39. Butler KV, Kalin J, Brochier C, Vistoli G, Langley B and Kozikowski AP: Rational design and simple chemistry yield a superior, neuroprotective HDAC6 inhibitor, tubastatin A. J Am Chem Soc 132: 10842-10846, 2010.

40. Tang W, Luo T, Greenberg EF, Bradner JE and Schreiber SL: Discovery of histone deacetylase 8 selective inhibitors. Bioorg Med Chem Lett 21: 2601-2605, 2011.

41. Kim JY, Park SJ, Yun KJ, Cho YW, Park HJ and Lee KT: Isoliquiritigenin isolated from the roots of Glycyrrhiza uralensis inhibits LPS-induced iNOS and COX-2 expression via the attenuation of NF-kappaB in RAW 264.7 macrophages. Eur J Pharmacol 584: 175-184, 2008.

42. Pandey MK, Sandur SK, Sung B, Sethi G, Kunnumakkara AB and Aggarwal BB: Butein, a tetrahydroxychalcone, inhibits nuclear factor (NF)-kappaB and NF-kappaB-regulated gene expression through direct inhibition of IkappaBalpha kinase beta on cysteine 179 residue. J Biol Chem 282: 17340-17350, 2007.

43. Orlikova B, Tasdemir D, Golais F, Dicato M and Diederich M: Dietary chalcones with chemopreventive and chemotherapeutic potential. Genes Nutr 6: 125-147, 2011. 